\title{
Erratum to: Sphingomonas panacis sp. nov., isolated from rhizosphere of rusty ginseng
}

\author{
Priyanka Singh • Yeon-Ju Kim • \\ Van-An Hoang • Mohamed El-Agamy Farh • \\ Deok-Chun Yang
}

Published online: 3 March 2016

(C) Springer International Publishing Switzerland 2016

\section{Erratum to: Antonie van Leeuwenhoek (2015) 108: 711-720 \\ DOI 10.1007/s10482-015-0527-y}

Subsequent to the publication of the above paper, it has been noticed that some minor details and the KCTC culture collection code given in the original protologue are incorrect. The corrected protologue is given below.

\section{Description of Sphingomonas panacis sp. nov.}

Sphingomonas panacis (pa'na.cis. N.L. gen. n. panacis of Panax ginseng, the host plant from which the type strain of this species was isolated).

The online version of the original article can be found under doi:10.1007/s10482-015-0527-y.

P. Singh · Y.-J. Kim $(\bowtie) \cdot$ V.-A. Hoang ·

D.-C. Yang $(\bowtie)$

Department of Oriental Medicine Biotechnology, Ginseng

Bank, College of Life Science, Kyung Hee University,

Seocheon-dong, Giheung-gu, Yongin-si,

Gyeonggi-do 446-701, Republic of Korea

e-mail: yeonjukim@khu.ac.kr

D.-C. Yang

e-mail: dcyang@khu.ac.kr

M. E.-A. Farh · D.-C. Yang

Graduate School of Biotechnology, College of Life

Science, Kyung Hee University, Yongin-si,

Gyeonggi-do 446-701, Republic of Korea
Gram-negative, aerobic, non-spore forming, motile and rod shaped, as well as positive for oxidase and catalase activity. Colonies are circular and dense, exhibiting a low convex shape with a smooth, opaque, light yellow appearance and a diameter of $0.1-1.0 \mathrm{~mm}$ after growth on NA plates for 1 day. Possesses lophotrichous flagella and grows well on TSA, R2A, $\mathrm{NA}, \mathrm{LB}$, and PDA agar. The temperature and $\mathrm{pH}$ range for growth is $10-37{ }^{\circ} \mathrm{C}$ and $\mathrm{pH} 5.5-7.5$ respectively. Growth occurs at $\mathrm{NaCl}$ (w:v) concentrations ranging from 0 to $1 \%$. Optimal growth occurs at $25{ }^{\circ} \mathrm{C}, \mathrm{pH}$ $6.0-6.5$, and in the presence of $1 \% \mathrm{NaCl}$. The bacterium is unable to hydrolyse carboxymethylcellulose, starch, DNAse, Tween 20, Tween 80 or casein. Positive for esculin hydrolysis and negative for indole, urease, nitrate reduction, and arginine dihydrolase activity. According to the API ZYM kit test, negative for cysteine arylamidase, trypsin, $\alpha$-chymotrypsin, $\alpha$ galactosidase, $\beta$-galactosidase, $\alpha$-glucosidase, $N$ acetyl- $\beta$-glucosaminidase, $\alpha$-mannosidase and $\alpha$-fucosidase activity. Positive for alkaline phosphatase, lipase (C14), leucine arylamidase, valine arylamidase, acid phosphatase, naphthol-AS-BI-phosphohydrolase, $\beta$-glucuronidase, $\beta$-glucosidase and weakly positive for esterase (C4) and esterase lipase (C8) activity. According to the API 20NE test, positive results of hydrolysis are obtained with assimilation of esculin, glucose, arabinose, mannose, mannitol, $\mathrm{N}$-acetyl-glucosamine, maltose, potassium gluconate, adipic acid, and malate; weakly positive for assimilation of $\beta$ galactosidase; and negative for fermentation of 
glucose, hydrolysis of gelatin, and assimilation of capric acid, trisodium citrate, and phenylacetic acid. According to the API ID32 GN kit test, positive for Lrhamnose, D-saccharose (sucrose), glycogen, L-serine, D-glucose and L-arabinose metabolism. Conversely, negative for $\mathrm{N}$-acetyl-glucosamine, D-ribose, myoinositol, D-maltose, itaconic acid, suberic acid, sodium malonate, sodium acetate, lactic acid, L-alanine, potassium 5-ketogluconate, 3-hydroxybenzoic acid, D-mannitol, salicin, D-melibiose, L-fucose, D-sorbitol, propionic acid, carpic acid, valeric acid, trisodium citrate, L-histidine, potassium 2-ketogluconate, 3-hydroxybutyric acid, 4-hydroxybenzoic acid, and Lproline metabolism. The DNA $\mathrm{G}+\mathrm{C}$ content of the type strain is $64.4 \mathrm{~mol} \%$, and the major cellular fatty acids are $\mathrm{C}_{14: 0} 2 \mathrm{OH}, \mathrm{C}_{16: 0}$ and summed feature $8\left(\mathrm{C}_{18: 1}\right.$ $\omega 7 \mathrm{c}$ and $\left.\mathrm{C}_{18: 1} \omega 6 \mathrm{c}\right)$. The major polar lipids are sphingoglycolipid, diphosphatidylglycerol, phosphatidylethanolamine, phosphatidylglycerol and phosphatidylcholine. The predominant isoprenoid quinone is ubiquinone Q-10. The major polyamine content is sym-homospermidine. Other biochemical characteristics are given in Table 2.

The type strain DCY99 ${ }^{\mathrm{T}}\left(=\mathrm{JCM} 30806^{\mathrm{T}}=\mathrm{KCTC}\right.$ $42347^{\mathrm{T}}=$ CCTCC AB $2015192^{\mathrm{T}}$ ) was isolated from the surface of rusty mountain ginseng at Hwacheon Mountain of Gangwon Province, Republic of Korea. The GenBank/EMBL/DDBJ accession number for the $16 \mathrm{~S}$ rRNA gene sequence of strain $\mathrm{DCY}^{\mathrm{T}}{ }^{\mathrm{T}}$ is KM819014. 